Asporin (ASPN) is differentially expressed in brain metastatic breast cancer.

Shahan Mamoor, MS ${ }^{1}$

1shahanmamoor@gmail.com

East Islip, NY USA

Breast cancer patients diagnosed with metastasis to the brain ${ }^{1-3}$ are presented limited treatment options ${ }^{4,5}$. We mined published microarray data 6,7 to discover genes associated with brain metastasis in human breast cancer, and identified differential expression of the gene encoding asporin, ASPN, when comparing brain metastatic tissues to primary tumors of the breast. ASPN was expressed at significantly lower levels in metastasis to the brain as compared to primary tumors, and primary tumor expression of ASPN was significantly correlated with overall survival ${ }^{8}$. Asporin ${ }^{9}$, its ability to interact with TGF- $\beta^{10}$ and type I collagen ${ }^{11}$, and inhibition of these interactions through down-regulation of asporin expression may be relevant to pathways underlying metastasis to the brain in human breast cancer.

Keywords: breast cancer, ASPN, brain metastasis, central nervous system metastasis, systems biology of breast cancer, targeted therapeutics in breast cancer. 
A study of patients treated with treated with trastuzumab, a monoclonal antibody targeting the human epidermal growth factor receptor 2 (HER2), reported a rate of metastasis to the central nervous system of $34 \%{ }^{2}$. The severity of disease and limited treatment options demand an enhanced understanding of the fundamental transcriptional nature of metastasis to the brain in patients with breast cancer. We mined published microarray data ${ }^{6,7}$ generated using the primary tumors and brain metastatic tissues of patients with human breast cancer to identify genes whose expression was most quantitatively different in brain metastatic tissues. These analyses revealed significant differential expression of ASPN in metastases to the brain in patients with human breast cancer.

Methods

We used datasets GSE438376 and GSE1259897 for this differential gene expression analysis of brain metastasis in human breast cancer in conjunction with GEO2R. GSE43837 was generated using Affymetrix Human X3P Array technology with $n=19$ primary breast tumors and $n=19$ brain metastases from patients with breast cancer, analysis performed using platform GPL1352. GSE125989 was generated using Affymetrix Human Genome U133A 2.0 Array technology with $n=16$ primary tumors from patients with breast cancer and $n=16$ brain metastases from patients with brain metastatic breast cancer. The Benjamini and Hochberg method of $p$-value adjustment was used for ranking of differential expression but raw $p$-values were used to assess statistical significance of global differential expression. Log-transformation of data was auto-detected, and the NCBI generated category of platform annotation was used. A statistical test was performed to evaluate whether ASPN gene expression was significantly between primary breast tumors and brain metastases in humans with breast cancer using a two-tailed, unpaired t-test with Welch's correction. We used PRISM for all statistical analyses of differential gene expression in human breast cancer (Version 8.4.0)(455). For Kaplan-Meier survival analysis, we used the Kaplan-Meier plotter online tool ${ }^{8}$ for correlation of ASPN mRNA expression levels with relapse

We performed comparative transcriptome analysis of primary and metastatic tumor tissues from patients with breast cancer using published microarray data 6,7 to describe the transcriptional landscape of brain metastasis in human breast cancer and for the identification of potential therapeutic targets.

\section{ASPN is differentially expressed in the brain metastases of patients with metastatic breast cancer} when compared to primary tumors of the breast.

We identified the gene encoding asporin, ASPN, as among the genes whose expression was most significantly different in the brain metastatic tissues of patients with breast cancer as compared to primary tumors of the breast. When sorting each of the genes expressed in brain metastases based on significance of change in expression as compared to primary tumors of the breast, ASPN ranked 12 out of 61359 total transcripts (Table 1), equating to $99.98 \%$ differential expression. Differential expression of ASPN in metastases to the brain was statistically significant (Table $1 ; p=0.00003002$ ).

We mined a second microarray dataset to validate differential expression of ASPN in brain metastatic breast cancer. We again identified ASPN as among the genes whose expression was most different in the brain metastatic tissues of patients with breast cancer as compared to primary tumors of the breast. When sorting each of the genes expressed in brain metastases based on significance of change in expression as compared to primary tumors of the breast, in this dataset, ASPN ranked 190 out of 22277 total transcripts (Table 2), equating to $99.1 \%$ differential expression. Differential expression of ASPN in metastases to the brain was again statistically significant (Table $2 ; p=1.35 \mathrm{E}-03$ ). 
ASPN is expressed at lower levels in metastases to the brain as compared to primary tumors of the breast.

We obtained exact mRNA expression values for ASPN from primary tumors of the breast and from brain metastases of patients with breast cancer to evaluate magnitude and direction of change in ASPN mRNA expression in metastasis to the brain. ASPN was expressed at lower levels in metastasis to the brain as compared to primary tumors of the breast (Figure 1), but this difference was not deemed statistically significant (Figure $1 ; p=0.1246$ ). We calculated a mean fold change of $0.22 \pm 0.88$ in ASPN mRNA expression in brain metastases relative to primary tumors of the breast (Table 1).

We also obtained exact mRNA expression values for ASPN from primary tumors of the breast and from brain metastases of patients with breast cancer from a second dataset to evaluate magnitude and direction of change in ASPN mRNA expression in metastasis to the brain. We again observed decreased expression of ASPN at lower levels in metastasis to the brain as compared to primary tumors of the breas (Figure 2), and this difference was statistically significant (Figure $2 ; p=0.0044$ ). We calculated a mean fold change of $0.80 \pm 0.19$ in ASPN mRNA expression in brain metastases relative to primary tumors of the breast (Table 2).

\section{ASPN expression associates with survival outcomes in breast cancer.}

We performed Kaplan-Meier survival analysis to determine whether ASPN mRNA expression levels correlated with survival outcomes in patients with breast cancer. In 1402 breast cancer patients, we observed a statistically significant correlation between ASPN expression and overall survival (OS) (Figure 3; log rank $p$-value: 0.024 for overall survival, hazard ratio: $0.78(0.63$ - 0.97) (Fig. 3)). Lower primary tumor ASPN expression, like which we observed in metastases to the brain, was a negative prognostic indicator in patients with breast cancer. Median OS was 81.6 months for patients with low tumor expression of ASPN while median OS was 120 months for patients with high tumor expression of ASPN (Table 3).

Thus, through analysis of published microarray datasets 6,7 , we found that ASPN was among the genes whose expression was most significantly different in the brain metastases of patients with metastatic breast cancer as compared to primary tumors of the breast, that ASPN expression was significantly lower in brain metastatic tissues as compared to primary tumors of the breast, and that low primary tumor expression of ASPN significantly correlated with worse overall survival as compared to patients whose tumors expressed high levels of ASPN.

\section{Discussion}

ASPN shares similarity at the genetic level with decorin and biglycan and was purified from cartilage and meniscus. Asporin, a member of the family of leucine-rich repeat molecules, contains 10 leucine-rich repeats, 4 amino-terminal cysteine and 2 carboxy-terminal cysteine residues, in addition to a propeptide, an amino-terminal stretch rich in aspartic acid residues, and an amino-terminal $\mathrm{N}$ glycosylation site 9 . Expression of aspirin was observed in articular cartilage of patients with osteoarthritis, aorta, the uterus, liver and the heart ${ }^{9}$. In articular cartilage, asporin can interact with the transforming growth factor beta (TGF- $\beta$ ) and impair chondrogenesis induced by TGF- $\beta{ }^{10}$. Another interaction partner of asporin is type I collagen; this interaction can be inhibited by decorin but not biglycan. The polyaspartate domain of asporin can influence mineralization of collagen, and expression of Osterix and Runx2, markers of the osteoblast, could be induced by asporin ${ }^{11}$.

We provided evidence here that asporin was among the genes most differentially expressed in metastases to the brain as compared to primary tumors of the breast, that asporin was expressed at significantly lower levels in brain metastatic tissues as compared to primary tumors of the breast, and primary tumor ASPN expression was significantly correlated with overall survival in patients with breast cancer. Functions of asporin, including binding to TGF- $\beta$, type I collagen, and its influence over chondrogenesis and collagen mineralization may be of relevance to the processes which control metastasis of tumor cells from the breast to the brain. 


\section{References}

1. Lin, N.U., Amiri-Kordestani, L., Palmieri, D., Liewehr, D.J. and Steeg, P.S., 2013. CNS metastases in breast cancer: old challenge, new frontiers.

2. Bendell, J.C., Domchek, S.M., Burstein, H.J., Harris, L., Younger, J., Kuter, I., Bunnell, C., Rue, M., Gelman, R. and Winer, E., 2003. Central nervous system metastases in women who receive trastuzumab-based therapy for metastatic breast carcinoma. Cancer, 97(12), pp.2972-2977.

3. Tsukada, Y., Fouad, A., Pickren, J.W. and Lane, W.W., 1983. Central nervous system metastasis from breast carcinoma autopsy study. Cancer, 52(12), pp.2349-2354.

4. Robson, M., Im, S.A., Senkus, E., Xu, B., Domchek, S.M., Masuda, N., Delaloge, S., Li, W., Tung, N., Armstrong, A. and Wu, W., 2017. Olaparib for metastatic breast cancer in patients with a germline BRCA mutation. New England Journal of Medicine, 377(6), pp.523-533.

5. Turner, N.C., Ro, J., André, F., Loi, S., Verma, S., Iwata, H., Harbeck, N., Loibl, S., Huang Bartlett, C., Zhang, K. and Giorgetti, C., 2015. Palbociclib in hormone-receptor-positive advanced breast cancer. New England Journal of Medicine, 373(3), pp.209-219.

6. McMullin, R.P., Wittner, B.S., Yang, C., Denton-Schneider, B.R., Hicks, D., Singavarapu, R., Moulis, S., Lee, J., Akbari, M.R., Narod, S.A. and Aldape, K.D., 2014. A BRCA1 deficient-like signature is enriched in breast cancer brain metastases and predicts DNA damage-induced poly (ADP-ribose) polymerase inhibitor sensitivity. Breast Cancer Research, 16(2), pp.1-10.

7. Iwamoto, T., Niikura, N., Ogiya, R., Yasojima, H., Watanabe, K.I., Kanbayashi, C., Tsuneizumi, M., Matsui, A., Fujisawa, T., Iwasa, T. and Shien, T., 2019. Distinct gene expression profiles between primary breast cancers and brain metastases from pair-matched samples. Scientific reports, 9(1), pp. $1-8$.

8. Györffy, B., Lanczky, A., Eklund, A.C., Denkert, C., Budczies, J., Li, Q. and Szallasi, Z., 2010. An online survival analysis tool to rapidly assess the effect of 22,277 genes on breast cancer prognosis using microarray data of 1,809 patients. Breast cancer research and treatment, 123(3), pp.725-731.

9. Lorenzo, P., Aspberg, A., Önnerfjord, P., Bayliss, M.T., Neame, P.J. and Heinegård, D., 2001. Identification and characterization of asporin a novel member of the leucine-rich repeat protein family closely related to decorin and biglycan. Journal of Biological Chemistry, 276(15), pp.12201-12211.

10.Nakajima, M., Kizawa, H., Saitoh, M., Kou, I., Miyazono, K. and Ikegawa, S., 2007. Mechanisms for asporin function and regulation in articular cartilage. Journal of biological chemistry, 282(44), pp. 32185-32192.

11.Kalamajski, S., Aspberg, A., Lindblom, K., Heinegård, D. and Oldberg, Å., 2009. Asporin competes with decorin for collagen binding, binds calcium and promotes osteoblast collagen mineralization. Biochemical Journal, 423(1), pp.53-59. 


\begin{tabular}{|c|c|c|c|c|c|c|c|}
\hline Rank & ID & $p$-value & $\mathbf{t}$ & B & FC & Gene & Gene name \\
\hline 190 & 219087_at & 1.35E-03 & -3.4879469 & -0.997 & $0.80 \pm 0.19$ & ASPN & asporin \\
\hline
\end{tabular}

Table 2: Differential expression of ASPN in brain metastatic tissues as compared to primary tumors in patients with breast cancer.

Rank of differential expression, probe ID, $p$-value with respect to differential expression, $\mathrm{t}$, a moderated tstatistic, B, the log-odds of differential expression between the two groups compared, gene and gene name are listed in this chart.

PAGE 6 


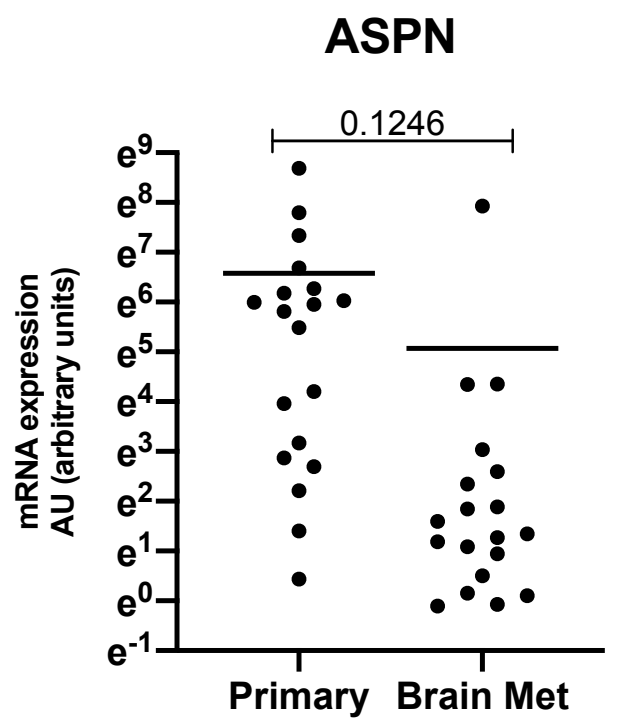

Figure 1: ASPN is expressed at lower levels in brain metastases as compared to primary tumors of the breast in metastatic breast cancer.

The mRNA expression level of ASPN in primary tumors of the breast (left) and in the brain metastases of brain metastatic breast cancer patients (right) is graphically represented here with mean ASPN levels marked and the result of a statistical test evaluating difference in mRNA expression level between brain metastases and primary tumors of the breast, a $p$-value, listed above. The Y-axis is set to natural logarithm (ln). 


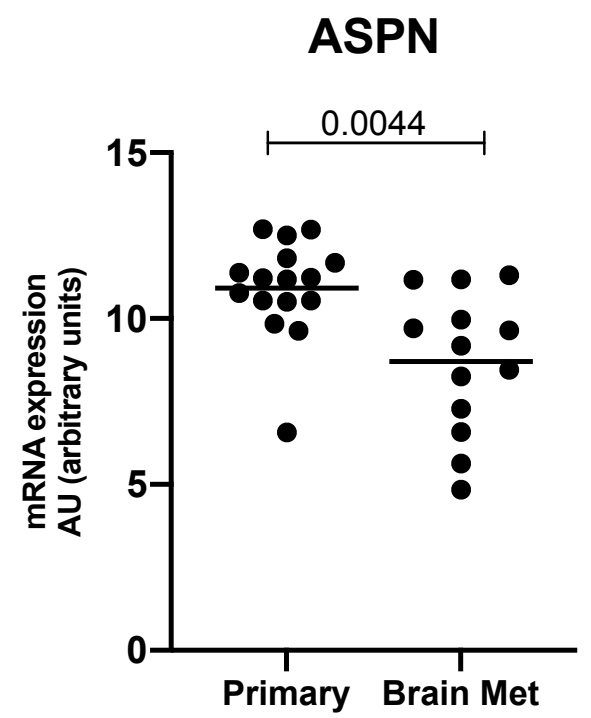

Figure 2: ASPN is expressed at significantly lower levels in brain metastases as compared to primary tumors of the breast in metastatic breast cancer.

The mRNA expression level of ASPN in primary tumors of the breast (left) and in the brain metastases of brain metastatic breast cancer patients (right) is graphically represented here with mean ASPN levels marked and the result of a statistical test evaluating difference in mRNA expression level between brain metastases and primary tumors of the breast, a $p$-value, listed above. The Y-axis is set to linear. 


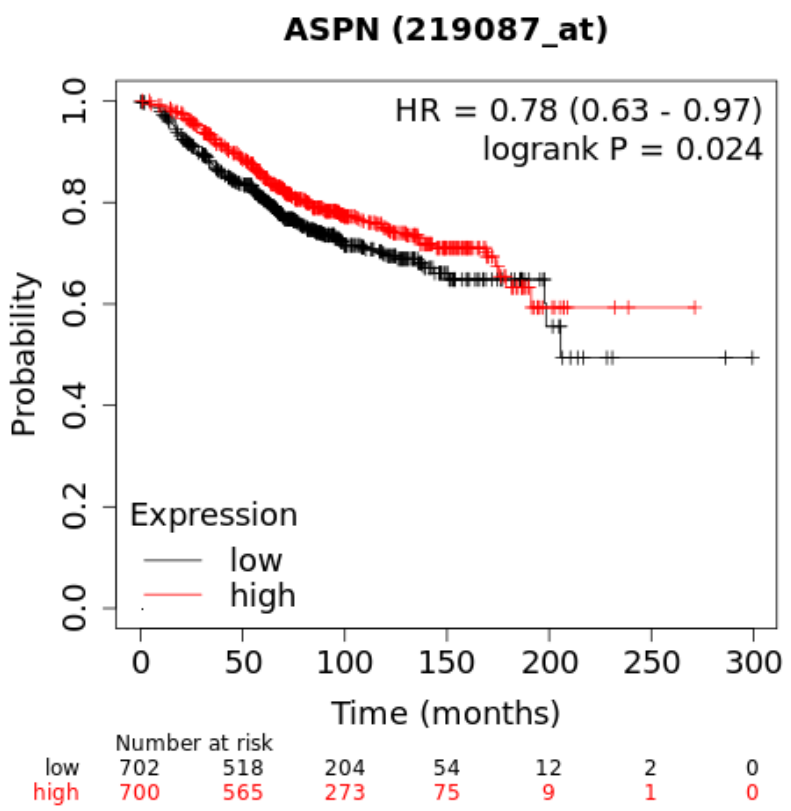

Figure 3: Expression of ASPN in primary tumors of the breast significantly correlates with overall survival in patients with breast cancer.

Depicted in this Kaplan-Meier plot is the probability of overall survival (OS) for $n=1402$ total patients stratified into two groups, based on low or high expression of ASPN in patient primary tumors. The log rank $p$-value denoting statistical significance of difference in overall survival when comparing the two groups, as well as hazard ratio for this comparison is listed above. Listed below is the number of patients at risk (number of patients alive) per interval, after stratification based on ASPN expression; in the first interval, number at risk is number of patients alive; in each subsequent interval, number at risk is the number at risk less those who have expired or are censored. 


\section{Low expression cohort (months) High expression cohort (months)}

81.6

120

Table 3: Median overall survival is greater in breast cancer patients whose primary tumors express high levels of ASPN.

The median overall survival of $n=1402$ breast cancer patients based on stratification into low or high tumor expression of ASPN is listed in this chart.

PAGE 10 